\title{
Current Pharmacological Advances in the Treatment of Cardiac Arrest
}

\author{
Andry Papastylianou and S. Mentzelopoulos \\ Intensive Care Unit, Evagelismos Hospital, 45-47 Ipsilantou Street, Athens 10676, Greece \\ Correspondence should be addressed to Andry Papastylianou, andry.papastylianou@gmail.com
}

Received 11 August 2011; Accepted 6 September 2011

Academic Editor: Michael Winters

Copyright ( 2012 A. Papastylianou and S. Mentzelopoulos. This is an open access article distributed under the Creative Commons Attribution License, which permits unrestricted use, distribution, and reproduction in any medium, provided the original work is properly cited.

Cardiac arrest is defined as the sudden cessation of spontaneous ventilation and circulation. Within 15 seconds of cardiac arrest, the patient loses consciousness, electroencephalogram becomes flat after 30 seconds, pupils dilate fully after 60 seconds, and cerebral damage takes place within $90-300$ seconds. It is essential to act immediately as irreversible damage can occur in a short time. Cardiopulmonary resuscitation (CPR) is an attempt to restore spontaneous circulation through a broad range of interventions which are early defibrillation, high-quality and uninterrupted chest compressions, advanced airway interventions, and pharmacological interventions. Drugs should be considered only after initial shocks have been delivered (when indicated) and chest compressions and ventilation have been started. During cardiopulmonary resuscitation, no specific drug therapy has been shown to improve survival to hospital discharge after cardiac arrest, and only few drugs have a proven benefit for short-term survival. This paper reviews current pharmacological treatment of cardiac arrest. There are three groups of drugs relevant to the management of cardiac arrest: vasopressors, antiarrhythmics, and other drugs such as sodium bicarbonate, calcium, magnesium, atropine, fibrinolytic drugs, and corticosteroids.

\section{Introduction}

Cardiac arrest constitutes a major health problem with dismal prognosis. Cardiac arrest may be caused either by asystole, pulseless electrical activity (PEA), pulseless ventricular tachycardia (VT), or ventricular fibrillation (VF) [1].

Data from 37 communities in Europe indicate that the annual incidence of Emergency Medical Services- (EMS-) treated, out-of-hospital, cardiopulmonary arrest for all rhythms is 38 per 100,000 population [2]. The annual incidence of EMS-treated VF arrest is 17 per 100,000. Survival to hospital discharge is $10.7 \%$ for all-rhythm and $21.2 \%$ for VF cardiac arrest. Recent data from 10 North American sites are consistent with these figures: median rate of survival to hospital discharge was $8.4 \%$ after EMS-treated cardiac arrest from any rhythm and $22.0 \%$ after VF [3].

In-hospital cardiac arrest occurs in 1 to 5 per 1000 patient admissions [4]. The American Heart Association's National Registry of cardiopulmonary resuscitation (CPR) indicate that survival to hospital discharge after in-hospital cardiac arrest is $17.6 \%$ (for all rhythms) [5]. The initial rhythm is VF or pulseless-VT in $25 \%$ of cases and, of these, $37 \%$ leave the hospital alive. After PEA or asystole, $11.5 \%$ survive to hospital discharge.

Multistage algorithms have been developed for CPR. In the algorithm for CPR, cardiac arrest rhythms are divided into two groups: shockable rhythms (VF/pulseless VT) and nonshockable rhythms (asystole, PEA).

The main difference in the treatment of these groups is the need for attempted defibrillation in those patients with $\mathrm{VF} /$ pulseless VT. Subsequent actions, including high-quality chest compressions with minimal interruptions, airway management and ventilation, venous access, administration of resuscitation drugs, the identification and correction of reversible factors (hypoxia, hypovolaemia, hypothermia, hypo-/ hyperkalaemia $(4 \mathrm{Hs})$, thrombosis-coronary or pulmonary, tamponade, toxins, tension pneumothorax (4Ts)), and the use of therapeutic hypothermia in comatose survivors are common to both groups [6]. 
We emphasize the importance of high-quality CPR (including chest compressions of adequate rate and depth, allowing complete chest recoil after each compression, minimizing interruptions in chest compressions and avoiding excessive ventilation), early defibrillation, and the use of therapeutic hypothermia in comatose survivors.

In any case, during CPR, vascular access, drug delivery, and advanced airway management should not cause significant interruptions in chest compressions or delay defibrillation.

In this paper, we summarize current experimental and clinical data on the efficacy and safety of drugs during CPR (vasopressors, antiarrhythmics, and other drugs such as sodium bicarbonate, calcium, magnesium, atropine, fibrinolytic drugs, and corticosteroids).

\section{Vasopressors}

Cardiac arrest is characterized by global ischemia, tissue hypoxia, and acidosis. CPR aims to improve the oxygen supplydemand ratio, in order to reverse tissue hypoxia.

Vasopressors are drugs that have a predominantly vasoconstrictive action on the peripheral vasculature, both arterial and venous. These drugs are used, during cardiac arrest, primarily to increase coronary and cerebral perfusion pressures and flows and thus facilitate ROSC.

Furthermore, during cardiac arrest, there is insufficient release of endogenous vasoconstrictors as part of the stress response, and this is another reason for using vasopressors. To date, there is no placebo-controlled study that shows that the routine use of any vasopressor at any stage during cardiac arrest increases neurologically intact survival to hospital discharge. However, there is evidence that vasopressors are associated with an increased rate of ROSC.

Epinephrine and vasopressin are the most commonly studied vasopressors in cardiac arrest. Historically, epinephrine has been used for the treatment of cardiac arrest for over 100 years [7]. However, vasopressin has been the focus of considerable research effort and has gained some popularity as a potential adjunct or alternative to epinephrine. There are no alternative vasopressors (norepinephrine, phenylephrine) with proven survival benefit relative to epinephrine $[8,9]$.

2.1. Epinephrine. Epinephrine is a powerful agonist at both $\alpha$-and $\beta$-Adrenergic Receptors. Stimulation of the $\beta$-receptor activates Gs-proteins, which in turn activate adenyl cyclase and thus lead to the generation of circular adenosine monophosphate (cAMP). In cardiac myocytes, this leads to increased intracellular $\mathrm{Ca}^{2+}$ concentration and contractility (inotropic effect). The stimulation of the $\beta$-receptor can also cause positive chronotropic effects (increased heart rate) and dromotropic effects (augmented conduction). In contrast, $\alpha 1$-receptor stimulation results in the activation of phospholipase $\mathrm{C}$, increased formation of inositol phosphates $\left(\mathrm{IP}_{3}\right.$, $\left.\mathrm{IP}_{4}\right)$ and diacyl glycerol, and smooth muscle contraction (Figure 1).

Epinephrine has been used for the treatment of cardiac arrest mostly for its $\alpha$-adrenergic effects which cause systemic vasoconstriction, and increase coronary and cerebral per-

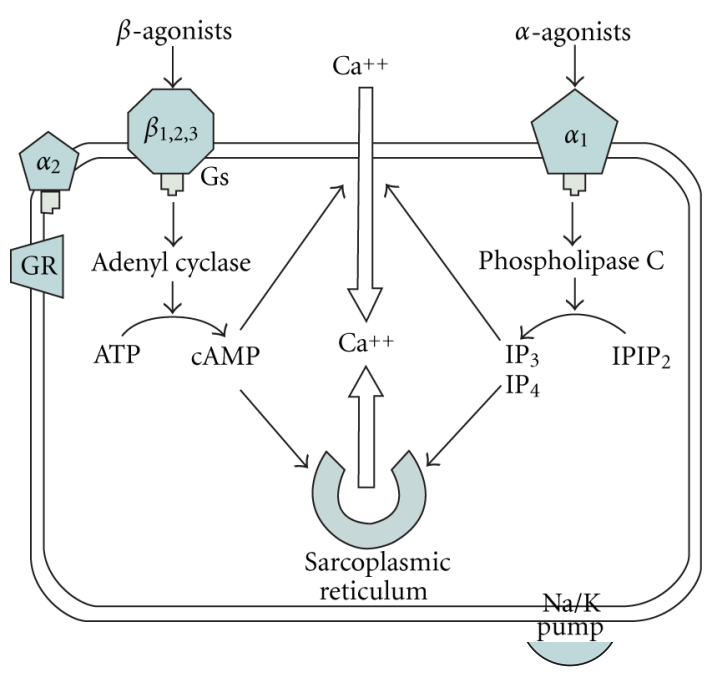

FIGURE 1: Schematic representation of the action of epinephrine on intracellular calcium in myocytes. ATP: adenosine triphosphate, cAMP: cyclic adenosine monophosphate, Gs: G protein complex, IP: inositol phosphate, $\mathrm{PIP}_{2}$ : phosphoinositol diphosphate, (adapted from $\mathrm{OH}$ 'S intensive care manual).

fusion pressures. The beta-adrenergic actions of adrenaline (inotropic, chronotropic) may increase coronary and cerebral blood flow, but may also have catastrophic effects such as the increase of myocardial oxygen consumption, ventricular arrhythmias (particularly when the cardiac tissue is acidotic), transient hypoxemia secondary to attenuation of hypoxic pulmonary vasoconstriction, and consequent increase in intrapulmonary, arteriovenous shunting, impaired microcirculation [10], and heart failure after ROSC [11]. Despite these deleterious effects and the limited evidence of benefit from the use of epinephrine during cardiac arrest, the improved short-term survival documented in some studies $[12,13]$ supports its continued use in cardiac arrest.

There are few data on the pharmacokinetics of epinephrine during CPR. In animal studies, peak plasma concentrations occur at about 90 seconds after a peripheral injection. The optimal dose of epinephrine is not known.

A meta-analysis of studies comparing standard- (1 mg or $0.02 \mathrm{mg} / \mathrm{kg}$ ) versus high dose (5-15 mg) epinephrine (3199 patients received high-dose and 3140 patients received standard dose), demonstrated improved ROSC in the high dose epinephrine group but failed to demonstrate any longterm survival benefit of the high and/or escalating dose of epinephrine [14]. Considering these results, Babbs et al. [15] concluded that a high initial dose of epinephrine in cardiac arrest may increase coronary perfusion pressures (CPP) and ROSC, but may also exacerbate postresuscitation myocardial dysfunction and cause harm. They concluded that high doses of epinephrine do not improve long-term survival and neurological outcome. Laboratory studies also documented adverse effects of high-dose epinephrine when given during $\mathrm{CPR}$, including postresuscitation hyperadrenergic state [16], myocardial necrosis [17], worsened postarrest cardiomyopathy [18], and greater early mortality relative to the standard dose [19]. 
In view of the above, current guidelines (2010) for ALS recommend a standard dose of $1 \mathrm{mg}$ of epinephrine (IV/IO) every 3 to 5 minutes during adult cardiac arrest (Class IIb, LOE A). Higher doses may be indicated to treat specific problems, such as a beta-blocker or calcium channel blocker overdose [6].

If the initial arrest rhythm is PEA or asystole, the administration of epinephrine is recommended as soon as vascular access is obtained. For VF or pulseless VT, the administration of epinephrine is recommended, after the third shock, and upon the resumption of chest compressions [20].

2.2. Vasopressin. The potentially catastrophic beta-effects of epinephrine led to the exploration of alternative vasopressors. In survivors of cardiac arrest endogenous vasopressin levels are higher than in nonsurvivors [21].

Arginine vasopressin (AVP) - a hypothalamic hormonealso known as antidiuretic hormone is a nonapeptide, that is, a 9 amino acid peptide. AVP is released from the posterior pituitary mostly when plasma osmolality is increased or plasma volume is reduced.

It acts via specific G-protein-coupled receptors. Three specific vasopressin receptors $\left(\mathrm{V}_{1,2,3}\right)$ are responsible for vasopressin's pharmacological effects. $V_{1}$ receptors are located in vascular smooth muscle and mediate vasoconstriction, $\mathrm{V}_{2}$ receptors are located in the distal convoluted tubules and medullary collecting ducts and mediate antidiuresis, $\mathrm{V}_{3}$ receptors are located in the anterior hypophysis and pancreatic isles and seem to affect insulin secretion, facilitate adrenocorticotropin release, and modulate memory, body temperature, and blood pressure.

Vasopressin is metabolized by vasopressinases in the liver and kidney and its half-life is $10-35 \mathrm{~min}$. It has several advantages over epinephrine in CPR because it improves cerebral and myocardial blood flow via $\mathrm{V}_{1}$-receptor mediated vasoconstriction, without the unwanted beta-effects of epinephrine. Also, in contrast to epinephrine, vasopressin's vasoconstrictive effect is preserved during hypoxia and severe acidosis. In addition, the risk of postresuscitation myocardial dysfunction is lower [22].

The effect of vasopressin during CPR has been extensively investigated in laboratory models. Animal studies demonstrated that vasopressin versus epinephrine increased blood flow in vital organs [23], improved cerebral oxygen delivery [24], and increased the probability of restoring spontaneous circulation [25] and neurologic outcome [26], as compared with epinephrine. The combination of vasopressin and epinephrine tripled coronary perfusion pressure versus either epinephrine or vasopressin alone, in a pig model of prolonged asphyxia [27]. In contrast to the animal studies, human trials showed no clear benefit of vasopressin over epinephrine in the treatment of cardiac arrest.

Three randomised controlled trials [28-30] and a metaanalysis [31] demonstrated no difference in outcomes (ROSC, survival to hospital discharge, or neurological outcome) between vasopressin and epinephrine used as first line vasopressor in cardiac arrest. Similarly, there was no evidence of harm from the use of vasopressin given during CPR.

Lindner et al. [28] conducted a randomized controlled trial involving 40 patients with out-of-hospital VF that was unresponsive to defibrillation. Vasopressin versus epinephrine increased 24-hour survival, but there was no betweengroup difference in the other outcomes (i.e., ROSC, survival on hospital admission, survival to hospital discharge, and neurological outcome).

Stiell et al. [29] conducted a randomized controlled trial involving 200 patients with in-hospital cardiac arrest and showed no statistically significant differences in outcomes (survival to 1 hour, survival to hospital discharge and neurological outcome) between vasopressin and epinephrine.

Wenzel et al. [30] conducted a large, multicenter, randomized, controlled study involving 1219 patients with outof-hospital cardiac arrest and showed no overall statistically significant differences in outcomes (ROSC, survival to hospital discharge, and neurological outcome) between the vasopressin and epinephrine groups. Subgroup analyses showed that the use of vasopressin in patients with asystole was associated with higher rates of hospital admission and survival to hospital discharge.

Two more recent, randomized, controlled trials [32, 33] showed no difference in outcomes (ROSC, survival to hospital discharge, and neurological outcome) when comparing epinephrine in combination with vasopressin versus epinephrine alone in cardiac arrest. In the large clinical trial in France, a total of 1442 patients with out-of-hospital cardiac arrest received a combination of epinephrine and vasopressin, and 1452 received epinephrine alone. The authors concluded that compared with epinephrine alone, the combination of vasopressin and epinephrine during ALS for outof-hospital cardiac arrest does not improve outcome [33].

A recent study reported that the combination of vasopressin and epinephrine during cardiopulmonary resuscitation increased end-tidal carbon dioxide and mean arterial blood pressure [34]. This improvement in these surrogate measures of vital-organ perfusion may have been the mechanism responsible for the subsequent, observed increase in short-term survival.

Mentzelopoulos et al. [35] conducted a single-center, prospective, randomized, double-blind, placebo-controlled trial, involving 100 patients with in hospital refractory cardiac arrest and studied the efficacy of combined vasopressinepinephrine during CPR with corticosteroid supplementation during and after CPR, compared with epinephrine alone, without corticosteroid supplementation. The authors concluded that compared with epinephrine alone, the combination of vasopressin and epinephrine with corticosteroid supplementation during and after CPR improves ROSC and survival to hospital discharge.

In view of the above, the American Heart Association current guidelines (2010) for ALS recommend that vasopressin (40 units) may replace either the first or the second dose of epinephrine in the treatment of cardiac arrest (Class IIb, LOE A).

\section{Antiarrhythmics}

Antiarrhythmic drugs produce pharmacologic effects by blocking sodium, potassium, and calcium ion channels present in the heart. The cardiac action potential is the result 
of multiple inward and outward ion currents with specific ion channels responsible for each of its five phases. The effects of antiarrhythmic drugs on the action potential and effective refractory period of the cardiac action potential determine the clinical effect of these drugs.

Lidocaine has traditionally been the antiarrhythmic drug of choice for the treatment of shock-resistant VF and for the prevention of VF recurrence after out of hospital cardiac arrest [36]. However, amiodarone has been shown to increase survival to hospital admission after out of hospital cardiac arrest when compared with placebo or lidocaine and is now recommended as the antiarrhythmic drug of choice for the treatment of refractory VF/VT. Lidocaine is indicated in refractory VF/VT when amiodarone is unavailable.

3.1. Amiodarone. Amiodarone is a potent antiarrhythmic agent with a complex electrophysiological and pharmacological profile. It is primarily a Vaughan Williams Class III agent; it acts by inhibiting the inward potassium current. It also blocks sodium and calcium channels and has antiadrenergic effects (noncompetitive blockade of alpha and beta receptors). It prolongs the duration of the action potential, increases the refractoriness of all cardiac tissue, and also prolongs the QT interval. Amiodarone is effective in suppressing both supraventricular and ventricular tachyarrhythmias.

Amiodarone exerts important cardiovascular effects. It dilates coronary arteries and increases coronary blood supply, and it causes peripheral arterial vasodilation and decreases systemic vascular resistance. Hypotension and bradycardia are the major acute adverse effects from amiodarone. They are related to the rate of infusion and to the solvent (polysorbate 80 and benzyl alcohol) which causes histamine release [37]. These adverse effects can be prevented by slowing the rate of infusion and can be treated with fluids and/or inotropic drugs. Recently, a new aqueous formulation of intravenous amiodarone, that is, relatively free from these adverse effects was approved for use in the United States [38]. An animal study on refractory VF noted that the combination of amiodarone and epinephrine administered together produced haemodynamics as good as epinephrine alone [39].

In two randomized, double-blind clinical trials, amiodarone has been shown to be superior to both placebo and lidocaine in improving survival to hospital admission for patients with out-of-hospital refractory VF/pulseless VT [ 40 , $41]$.

The ARREST trial involved 504 patients randomized to receive either amiodarone $(300 \mathrm{mg}$ bolus after the failure of three shocks and administration of epinephrine) or placebo; $44 \%$ of amiodarone-treated patients and $34 \%$ of placebotreated patients survived to hospital admission $(P=0.03)$ [40].

The ALIVE trial involved 347 patients randomized to either amiodarone (initial bolus dose of $5 \mathrm{mg} / \mathrm{Kg}$ followed, if needed, by a second dose of $2.5 \mathrm{mg} / \mathrm{Kg}$ ) or lidocaine (initial bolus dose of $1.5 \mathrm{mg} / \mathrm{Kg}$, repeated once, if needed); more patients treated with amiodarone survived to hospital admission, compared with patients treated with lidocaine (23\% versus $12 \%, P<0.005)$ [41].
In view of the above, the American Heart Association current guidelines (2010) for ALS recommend that amiodarone may be considered for refractory VF/pulseless VT (Class IIb, LOE B), with an initial dose of $300 \mathrm{mg}$, which can be followed by one dose of $150 \mathrm{mg}$.

3.2. Lidocaine. Lidocaine, long considered an important antiarrhythmic drug for refractory VF/pulseless VT, is now relegated to a second-choice option. It decreases ventricular automaticity. It also has a sodium channel-blocking effect, which is increased in myocardial ischaemia. Signs of lidocaine toxicity such as paraesthesia, confusion, and convulsions may occur in a dose-related manner. It is considered that a safe dose of lidocaine must not exceed $3 \mathrm{mg} / \mathrm{Kg}$ over the first hour of administration.

In refractory VF/pulseless VT, lidocaine may be considered if amiodarone is not available (Class IIb, LOE B). The initial dose is $100 \mathrm{mg}(1-1.5 \mathrm{mg} / \mathrm{Kg})$ with additional bolus(es) of $50 \mathrm{mg}$ up to the maximum dose of $3 \mathrm{mg} / \mathrm{Kg}$.

3.3. Magnesium. Magnesium is a cofactor for many enzyme systems, including the myocardial sodium/potassium ATPase. It is an emerging antiarrhythmic agent that may be best classified as a sodium/potassium pump agonist. Magnesium has many electrophysiological effects, including blocking atrial L and $\mathrm{T}$ type calcium channels. It prolongs both atrial refractory period and conduction, inhibits potassium entry, and suppresses ventricular after-depolarizations.

The electrophysiological effects of magnesium are more potent in the presence of increased extracellular potassium, and thus the usefulness of magnesium seems to be greater in the presence of ischemia, where loss of potassium from the cell is a major consequence.

Intravenous magnesium sulphate is indicated in torsades de pointes associated with prolonged QT interval, ventricular, or supraventricular tachycardia associated with hypomagnesaemia, and digoxin toxicity.

In two studies, magnesium sulphate has been shown to facilitate termination of torsades de pointes associated with a prolonged QT interval $[42,43]$. A bolus dose of 1 to $2 \mathrm{~g}$ of magnesium sulphate diluted in $10 \mathrm{~mL}$ of $5 \%$ dextrose in water is recommended (Class IIb, LOE C).

It was assumed that magnesium might exert beneficial effects in cardiac arrest, mainly due to its antiarrhythmic and calcium-channel blocking properties [44]. However, three randomised controlled trials [45-47], have failed to demonstrate any benefit of giving magnesium routinely during cardiac arrest.

In view of the above, the American Heart Association guidelines for ALS (2010) do not recommend the routine administration of magnesium sulfate in cardiac arrest (Class III, LOE A), unless torsades de pointes is present.

\section{Other Drugs}

There is no evidence that the routine administration of other drugs such as atropine, calcium, sodium bicarbonate, or fibrinolytic drugs during cardiac arrest increases survival to 
hospital discharge. The administration of these drugs may be considered in specific cases of cardiac arrest.

4.1. Atropine. Atropine is an anticholinergic drug, an ester of tropic acid and tropine. It blocks muscarinic acetylcholine receptors, and, thus, it blocks the effect of the vagus nerve on both the sinoatrial node and the atrioventricular node, and thus increases sinus node automaticity and facilitates atrioventricular conduction.

Atropine is administered in doses of $0.6-3.0 \mathrm{mg}$ IV to counteract bradycardia in the presence of hypotension and to prevent the bradycardia associated with vagal stimulation. Side effects of atropine are dose-related. A dose of $\leq 0.5 \mathrm{mg}$ may produce an agonist action with a resulting bradycardia. The side effects of atropine are delirium, tachycardia, increase in cardiac work and ventricular arrhythmias, coma, hot skin, blurred vision, and/or urinary retention, pupillary muscle dilation.

The effect of atropine during CPR has been questioned.

In 1979, Brown et al. [48] noted the possible role of the parasympathetic nervous system in cardiac arrest and reported that atropine is beneficial during cardiac arrest. Due to this report, the AHA included atropine in the treatment of asystole.

In 1981, Coon et al. [49] failed to demonstrate any benefit from the administration of atropine in out-of-hospital asystole or PEA.

Stueven and colleagues [50] reported that patients who received atropine had better resuscitation rates and better survival when atropine was given late during ALS. Considering that there was no record of the rhythm at this point, we cannot exclude the possibility that patients had begun to regain a pulse when atropine was given. Recent studies have failed to show any benefit from the use of atropine in out of hospital or in hospital cardiac arrest [51-53].

Atropine is no longer included in the ALS algorithm.

4.2. Calcium. Calcium ions are involved in cellular excitation, excitation-contraction coupling, and muscle contraction in cardiac, skeletal, and smooth muscle cells. Increased extracellular calcium increases intracellular calcium concentrations, and the force of contraction of cardiac myocytes and vascular smooth muscle cells.

There is a direct correlation between duration of resuscitation, low serum ionized calcium levels and mortality. However, high plasma calcium concentration after exogenous calcium injection may promote myocardial damage and impair neurological recovery. Improved survival was suggested by case reports of calcium use in children during cardiac surgery [54]. However, studies of calcium during cardiac arrest failed to demonstrate any beneficial effect on survival [55-57].

Calcium is not recommended for the treatment of cardiac arrest (Class III, LOE B). Administration of calcium during resuscitation is considered only in specific circumstances such as hyperkalaemia, hypocalcaemia, and overdose of calcium channel-blocking drugs.

4.3. Sodium Bicarbonate. During cardiac arrest and CPR, combined respiratory and metabolic acidosis arises from the carbon dioxide retention as pulmonary gas exchange ceases and from the reduction in cellular oxygen availability which leads to the development of anaerobic metabolism with lactic acidosis. Severe acidosis inhibits myocardial contractility and also reduces the responsiveness to catecholamines.

Before 1986, sodium bicarbonate was routinely used during CPR, even without knowledge of the patient's acid-base status. This practice was changed due to the potential adverse effects of buffer therapy and due to the clinical studies that failed to demonstrate any advantage of using sodium bicarbonate during cardiac arrest [58-60].

Buffer therapy during CPR can exacerbate intracellular acidosis since it generates carbon dioxide that freely diffuses across cellular membranes. It is estimated that $1 \mathrm{mEq} / \mathrm{kg}$ of sodium bicarbonate, given intravenously, produces approximately $180 \mathrm{~mL}$ of carbon dioxide, requiring a transient doubling of alveolar ventilation to prevent hypercarbia. Sodium bicarbonate during CPR may also cause hypernatraemia and hyperosmolality to an already compromised circulation and brain, metabolic alkalosis and a leftward shift in the oxygen haemoglobin saturation curve, which further inhibits the release of oxygen to the tissues. Mild acidaemia causes vasodilation and thus increases cerebral blood flow.

The routine administration of sodium bicarbonate is not recommended in patients with cardiac arrest (Class III, LOE B). Sodium bicarbonate may be given in cases of tricyclic antidepressant overdose and hyperkalaemia.

4.4. Fibrinolytic Drugs. Thrombolytic therapy during CPR has two major effects. First, it can be effective in acute myocardial infarction (AMI) or massive pulmonary embolism $(\mathrm{PE})$, which are common causes of cardiac arrest. Second, it may improve microcirculatory reperfusion after ROSC. Cardiac arrest and CPR are associated with a marked activation of coagulation without adequate fibrinolysis [61]. The formation of microthrombi impairs microcirculation ("noreflow" phenomenon) and contributes to cerebral dysfunction. Thrombolytic therapy can dissolve intravascular blood clots and improve cerebral microcirculation [62].

CPR had been considered in the past as a relative contraindication for thrombolysis because of the potential risk for bleeding complications in association with chest compressions. However, currently available data do not confirm the aforementioned speculation [63], and ongoing CPR is not a contraindication to thrombolysis.

Case reports where thrombolysis was used as successful "last resort" therapy (i.e., when the sole alternative would be CPR termination [64]) were followed by several studies suggesting that thrombolysis during CPR benefits patients with pulmonary embolism, or acute myocardial infarction, or those who had been unresponsive to conventional resuscitation efforts [65-72].

Conversely, two large clinical trials failed to show any benefit in outcome with fibrinolytic therapy during CPR $[73,74]$.

Abu-Laban et al. [73] conducted a randomized, doubleblind, placebo-controlled trial on out-of-hospital thrombolysis during cardiac arrest. Study participants had PEA, and in more than one-third of them the collapse was unwitnessed. 
The authors found no evidence of thrombolysis benefit. The study was criticized mainly for its limited statistical power.

A multicenter European study [74] investigated whether thrombolysis with the use of tenecteplase during CPR improves survival in witnessed out-of-hospital cardiac arrest of presumed cardiac origin. The results showed no improvement in survival. Despite these negative results, the authors argued that their findings do not suggest that thrombolysis should be withheld in patients with cardiac arrest, if the primary pathologic condition is known to be responsive to such treatment.

Thrombolytic therapy should not be routinely used in cardiac arrest (Class III, LOE B). The administration of thrombolytic therapy may be considered on an "empirical basis," when pulmonary embolism is presumed or known to be the cause of cardiac arrest (Class IIa, LOE B). In such cases, CPR should be performed for at least 60-90 min before ending the resuscitation attempts $[63,75]$.

4.5. Corticosteroids. Relative to other stress states, cardiac arrest is associated with lower cortisol levels during and after CPR $[76,77]$. This adrenocortical dysfunction results in hypotension and shock.

"Postresuscitation disease," first described by Negovsky [78], shares common features with sepsis such as reversible myocardial dysfunction, vasodilatation, coagulopathy, and plasma cytokine elevation [76].

Glucocorticoids modulate vascular reactivity to catecholamines $[79,80]$ and decrease the production of vasodilators such as nitric oxide [81].

Most studies suggest that serum cortisol levels are higher in survivors of cardiac arrest [82] and low serum cortisol levels are associated with early postresuscitation mortality $[83,84]$.

In a prospective, nonrandomized, open-labeled clinical trial, patients receiving hydrocortisone during resuscitation had a significantly higher ROSC rate than those receiving placebo. In this study there was no significant difference between the two groups in terms of short-term survival and hospital discharge [85].

In a prospective, randomized, double-blind, placebocontrolled trial, the combination of vasopressin-epinephrine-methylprednisolone during CPR followed by hydrocortisone, when post-ROSC shock was present, was compared to epinephrine and placebo. The authors concluded that combination therapy increased ROSC and survival to hospital discharge [35].

Further studies are needed to investigate the effect of corticosteroid supplementation during and after CPR in order to cover some knowledge gaps in this field.

\section{Conclusion}

For CPR, we emphasize the importance of high-quality chest compressions (rate, depth, recoil), with minimum interruptions and early defibrillation when appropriate. In any case, drug delivery should not cause significant interruptions in these interventions.
No drug has been definitively shown to improve survival to hospital discharge after cardiac arrest. However, evaluation of drugs is difficult after prolonged ischemia time, which anyway minimizes the probability of survival. Future studies might benefit from applying an upper limit for the time from collapse to the administration of drugs (e.g., 5-10 min), in order to avoid the inclusion of potentially "futile cases."

Adrenaline remains the drug of choice during cardiac resuscitation and other drugs such as atropine, sodium bicarbonate, calcium, magnesium and fibrinolytic drugs may be considered only in specific circumstances.

Corticosteroids during and after CPR seem to confer benefits with respect to hemodynamics, intensity of postresuscitation systemic inflammatory response and organ dysfunction.

Further studies are needed to investigate the effect of corticosteroid supplementation during and after CPR and the effect of the combination of adrenaline and vasopressin during cardiac resuscitation in order to cover some knowledge gaps in the field.

\section{References}

[1] D. P. Zipes and H. J. Wellens, "Sudden cardiac death," Circulation, vol. 98, no. 21, pp. 2334-2351, 1998.

[2] C. Atwood, M. S. Eisenberg, J. Herlitz, and T. D. Rea, "Incidence of EMS-treated out-of-hospital cardiac arrest in Europe," Resuscitation, vol. 67, no. 1, pp. 75-80, 2005.

[3] G. Nichol, E. Thomas, C. W. Callaway et al., "Regional variation in out-of-hospital cardiac arrest incidence and outcome," Journal of the American Medical Association, vol. 300, no. 12, pp. 1423-1431, 2008.

[4] C. Sandroni, J. Nolan, F. Cavallaro, and M. Antonelli, "Inhospital cardiac arrest: incidence, prognosis and possible measures to improve survival," Intensive Care Medicine, vol. 33, no. 2, pp. 237-245, 2007.

[5] P. A. Meaney, V. M. Nadkarni, K. B. Kern, J. H. Indik, H. R. Halperin, and R. A. Berg, "Rhythms and outcomes of adult in-hospital cardiac arrest," Critical Care Medicine, vol. 38, no. 1, pp. 101-108, 2010.

[6] R. W. Neumar, C. W. Otto, M. S. Link et al., “2010 American heart association guidelines for cardiopulmonary resuscitation and emergency cardiovascular care science part 8: adult advanced cardiovascular life support," Circulation, vol. 122, no. 3, pp. S729-S767, 2010.

[7] R. Gottlieb, "Ueber die wirkung der nebennieren extracte auf herz und blutdruck," Archiv für Experimentelle Pathologie und Pharmakologie, vol. 38, no. 1-2, pp. 99-112, 1897.

[8] M. Callaham, C. D. Madsen, C. W. Barton, C. E. Saunders, and J. Pointer, "A randomized clinical trial of highdose epinephrine and norepinephrine vs standard-dose epinephrine in prehospital cardiac arrest," Journal of the American Medical Association, vol. 268, no. 19, pp. 2667-2672, 1992.

[9] T. Silfvast, L. Saarnivaara, and A. Kinnunen, "Comparison of adrenaline and phenylephrine in out-of-hospital cardiopulmonary resuscitation. A double-blind study," Acta Anaesthesiologica Scandinavica, vol. 29, no. 6, pp. 610-613, 1985.

[10] M. Fries, W. Tang, Y. T. Chang, J. Wang, C. Castillo, and M. H. Weil, "Microvascular blood flow during cardiopulmonary resuscitation is predictive of outcome," Resuscitation, vol. 71, no. 2, pp. 248-253, 2006. 
[11] M. G. Angelos, R. L. Butke, A. R. Panchal et al., "Cardiovascular response to epinephrine varies with increasing duration of cardiac arrest," Resuscitation, vol. 77, no. 1, pp. 101-110, 2008.

[12] T. M. Olasveengen, K. Sunde, C. Brunborg, J. Thowsen, P. A. Steen, and L. Wik, "Intravenous drug administration during out-of-hospital cardiac arrest: a randomized trial," Journal of the American Medical Association, vol. 302, no. 20, pp. 22222229, 2009.

[13] J. Herlitz, L. Ekstrom, B. Wennerblom, A. Axelsson, A. Bang, and S. Holmberg, "Adrenaline in out-of-hospital ventricular fibrillation. Does it make any difference?" Resuscitation, vol. 29, no. 3, pp. 195-201, 1995.

[14] C. Vandycke and P. Martens, "High dose versus standard dose epinephrine in cardiac arrest-a meta- analysis," Resuscitation, vol. 45, no. 3, pp. 161-166, 2000.

[15] C. F. Babbs, R. A. Berg, F. Kette et al., "Use of pressors in the treatment of cardiac arrest," Annals of Emergency Medicine, vol. 37, no. 4, pp. S152-S162, 2001.

[16] U. Hornchen, C. Lussi, and J. Schuttler, "Potential risks of high-dose epinephrine for resuscitation from ventricular fibrillation in a porcine model," Journal of Cardiothoracic and Vascular Anesthesia, vol. 7, no. 2, pp. 184-187, 1993.

[17] R. Neumar, N. Bircher, M. Radovsky et al., "Myocardial necrosis after high dose epinephrine during CPR," Annals of Emergency Medicine, vol. 22, pp. 892-893, 1993.

[18] W. Tang, M. H. Weil, S. Sun, M. Noc, L. Yang, and R. J. Gazmuri, "Epinephrine increases the severity of postresuscitation myocardial dysfunction," Circulation, vol. 92, no. 10, pp. 3089 3093, 1995.

[19] R. A. Berg, C. W. Otto, K. B. Kern et al., "High-dose epinephrine results in greater early mortality after resuscitation from prolonged cardiac arrest in pigs: a prospective, randomized study," Critical Care Medicine, vol. 22, no. 2, pp. 282-290, 1994.

[20] J. P. Nolan, J. Soar, D. A. Zideman et al., "European resuscitation council guidelines for resuscitation 2010 section 1 . Executive summary," Resuscitation, vol. 81, no. 10, pp. 1219 1276, 2010.

[21] K. H. Lindner, T. Haak, A. Keller, U. Bothner, and K. G. Lurie, "Release of endogenous vasopressors during and after cardiopulmonary resuscitation," Heart, vol. 75, no. 2, pp. 145150, 1996.

[22] D. K. Sreevastava, P. K. Roy, S. K. Dass et al., "Cardiopulmonary resuscitation: an overview of recent advances in concepts and practices," Medical Journal Armed Forces India, vol. 60, no. 1, pp. 52-58, 2004.

[23] K. H. Lindner, A. W. Prengel, E. G. Pfenninger et al., "Vasopressin improves vital organ blood flow during closed-chest cardiopulmonary resuscitation in pigs," Circulation, vol. 91, no. 1, pp. 215-221, 1995.

[24] A. W. Prengel, K. H. Lindner, and A. Keller, "Cerebral oxygenation during cardiopulmonary resuscitation with epinephrine and vasopressin in pigs," Stroke, vol. 27, no. 7, pp. 1241-1248, 1996.

[25] V. Wenzel, K. H. Lindner, A. C. Krismer, E. A. Miller, W. G. Voelckel, and W. Lingnau, "Repeated administration of vasopressin but not epinephrine maintains coronary perfusion pressure after early and late administration during prolonged cardiopulmonary resuscitation in pigs," Circulation, vol. 99, no. 10, pp. 1379-1384, 1999.

[26] V. Wenzel, K. H. Lindner, A. C. Krismer et al., "Survival with full neurologic recovery and no cerebral pathology after prolonged cardiopulmonary resuscitation with vasopressin in pigs," Journal of the American College of Cardiology, vol. 35, no. 2, pp. 527-533, 2000.
[27] V. D. Mayr, V. Wenzel, W. G. Voelckel et al., "Developing a vasopressor combination in a pig model of adult asphyxial cardiac arrest," Circulation, vol. 104, no. 14, pp. 1651-1656, 2001.

[28] K. H. Lindner, B. Dirks, H. U. Strohmenger, A. W. Prengel, I. M. Lindner, and K. G. Lurie, "Randomised comparison of epinephrine and vasopressin in patients with out-of-hospital ventricular fibrillation," The Lancet, vol. 349, no. 9051, pp. 535-537, 1997.

[29] I. G. Stiell, P. C. Hebert, G. A. Wells et al., "Vasopressin versus epinephrine for inhospital cardiac arrest: a randomised controlled trial," The Lancet, vol. 358, no. 9276, pp. 105-109, 2001.

[30] V. Wenzel, A. C. Krismer, H. R. Arntz, H. Sitter, K. H. Stadlbauer, and K. H. Lindner, "A comparison of vasopressin and epinephrine for out-of-hospital cardiopulmonary resuscitation," The New England Journal of Medicine, vol. 350, no. 2, pp. 105-113, 2004.

[31] K. Aung and T. Htay, "Vasopressin for cardiac arrest: a systematic review and meta-analysis," Archives of Internal Medicine, vol. 165, no. 1, pp. 17-24, 2005.

[32] C. W. Callaway, D. Hostler, A. A. Doshi et al., "Usefulness of vasopressin administered with epinephrine during out-ofhospital cardiac arrest," American Journal of Cardiology, vol. 98, no. 10, pp. 1316-1321, 2006.

[33] P. Y. Gueugniaud, J. S. David, E. Chanzy et al., "Vasopressin and epinephrine vs. epinephrine alone in cardiopulmonary resuscitation," The New England Journal of Medicine, vol. 359, no. 1, pp. 21-30, 2008.

[34] S. Mally, A. Jelatancev, and S. Grmec, "Effects of epinephrine and vasopressin on end-tidal carbon dioxide tension and mean arterial blood pressure in out-of-hospital cardiopulmonary resuscitation: an observational study," Critical Care, vol. 11, no. 2, Article ID R39, 2007.

[35] S. D. Mentzelopoulos, S. G. Zakynthinos, M. Tzoufi et al., "Vasopressin, epinephrine, and corticosteroids for in-hospital cardiac arrest," Archives of Internal Medicine, vol. 169, no. 1, pp. 15-24, 2009.

[36] "Guidelines 2000 for cardiopulmonary resuscitation and emergency cardiovascular care: an international consensus on science. 6. Advanced cardiovascular life support. 5. Pharmacology I: agents for arrhythmias," Circulation, vol. 102, supplement 1, pp. 112-128, 2000.

[37] E. Masini, J. Planchenault, F. Pezziardi, P. Gautier, and J. P. Gagnol, "Histamine-releasing properties of Polysorbate 80 in vitro and in vivo: correlation with its hypotensive action in the dog," Agents and Actions, vol. 16, no. 6, pp. 470-477, 1985.

[38] J. C. Somberg, S. Timar, S. J. Bailin et al., "Lack of a hypotensive effect with rapid administration of a new aqueous formulation of intravenous amiodarone," American Journal of Cardiology, vol. 93, no. 5, pp. 576-581, 2004.

[39] E. F. Paiva, M. B. Perondi, K. B. Kern et al., "Effect of amiodarone on haemodynamics during cardiopulmonary resuscitation in a canine model of resistant ventricular fibrillation," Resuscitation, vol. 58, no. 2, pp. 203-208, 2003.

[40] P. J. Kudenchuk, L. A. Cobb, M. K. Copass et al., "Amiodarone for resuscitation after out-of-hospital cardiac arrest due to ventricular fibrillation," The New England Journal of Medicine, vol. 341, no. 12, pp. 871-878, 1999.

[41] P. Dorian, D. Cass, B. Schwartz, R. Cooper, R. Gelaznikas, and A. Barr, "Amiodarone as compared with lidocaine for shockresistant ventricular fibrillation," The New England Journal of Medicine, vol. 346, no. 12, pp. 884-890, 2002.

[42] M. Manz, D. Pfeiffer, W. Jung, and B. Lueritz, "Intravenous treatment with magnesium in recurrent persistent ventricular 
tachycardia," New Trends in Arrhythmias, vol. 7, no. 4, pp. 437442, 1991.

[43] D. Tzivoni, S. Banai, C. Schuger et al., "Treatment of torsade de pointes with magnesium sulfate," Circulation, vol. 77, no. 2, pp. 392-397, 1988.

[44] M. A. Arsenian, "Magnesium and cardiovascular disease," Progress in Cardiovascular Diseases, vol. 35, no. 4, pp. 271-310, 1993.

[45] J. Allegra, R. Lavery, R. Cody et al., "Magnesium sulfate in the treatment of refractory ventricular fibrillation in the prehospital setting," Resuscitation, vol. 49, no. 3, pp. 245-249, 2001.

[46] M. C. Thel, A. L. Armstrong, S. E. McNulty, R. M. Califf, and C. M. O'Connor, "Randomised trial of magnesium in in-hospital cardiac arrest. Duke internal medicine housestaff," The Lancet, vol. 350, pp. 1272-1276, 1997.

[47] D. M. Fatovich, D. A. Prentice, and G. J. Dobb, "Magnesium in cardiac arrest (the magic trial)," Resuscitation, vol. 35, no. 3, pp. 237-241, 1997.

[48] D. C. Brown, A. J. Lewis, and J. M. Criley, "Asystole and its treatment: the possible role of the parasympathetic nervous system in cardiac arrest," Journal of the American College of Emergency Physicians, vol. 8, no. 11, pp. 448-452, 1979.

[49] G. A. Coon, J. E. Clinton, and E. Ruiz, "Use of atropine for brady asystolic prehospital cardiac arrest," Annals of Emergency Medicine, vol. 10, no. 9, pp. 462-467, 1981.

[50] H. A. Stueven, D. J. Tonsfeldt, B. M. Thompson, J. Whitcomb, E. Kastenson, and C. Aprahamian, "Atropine in asystole: human studies," Annals of Emergency Medicine, vol. 13, no. 9, part 2, pp. 815-817, 1984.

[51] J. Engdahl, A. Bang, J. Lindqvist, and J. Herlitz, "Can we define patients with no and those with some chance of survival when found in asystole out of hospital?" American Journal of Cardiology, vol. 86, no. 6, pp. 610-614, 2000.

[52] I. G. Stiell, G. A. Wells, B. Field et al., "Advanced cardiac life support in out-of-hospital cardiac arrest," The New England Journal of Medicine, vol. 351, no. 7, pp. 647-656, 2004.

[53] A. J. Tortolani, D. A. Risucci, S. R. Powell, and R. Dixon, "In-hospital cardiopulmonary resuscitation during asystole. Therapeutic factors associated with 24-hour survival," Chest, vol. 96, no. 3, pp. 622-626, 1989.

[54] J. H. Kay and A. Blalock, "The use of calcium chloride in the treatment of cardiac arrest in patients," Surgery, Gynecology \& Obstetrics, vol. 93, no. 1, pp. 97-102, 1951.

[55] E. E. Harrison and B. D. Amey, "The use of calcium in cardiac resuscitation," American Journal of Emergency Medicine, vol. 1, no. 3, pp. 267-273, 1983.

[56] H. A. Stueven, B. Thompson, C. Aprahamian, D. J. Tonsfeldt, and E. H. Kastenson, "Lack of effectiveness of calcium chloride in refractory asystole," Annals of Emergency Medicine, vol. 14, no. 7, pp. 630-632, 1985.

[57] H. A. Stueven, B. M. Thompson, C. Aprahamian, and D. J. Tonsfeldt, "Calcium chloride: reassessment of use in asystole," Annals of Emergency Medicine, vol. 13, no. 9, pp. 820-822, 1984.

[58] T. Dybvik, T. Strand, and P. A. Steen, "Buffer therapy during out-of-hospital cardiopulmonary resuscitation," Resuscitation, vol. 29, no. 2, pp. 89-95, 1995.

[59] T. P. Aufderheide, D. R. Martin, D. W. Olson et al., "Prehospital bicarbonate use in cardiac arrest: a 3-year experience," American Journal of Emergency Medicine, vol. 10, no. 1, pp. 47, 1992.

[60] H. Delooz and P. J. Lewi, "Are inter-center differences in EMS-management and sodium-bicarbonate administration important of the outcome of CPR? The cerebral resuscitation study group," Resuscitation, vol. 17, supplement, pp. S161S172, 1989.

[61] B. W. Bottiger, J. Motsch, H. Bohrer et al., "Activation of blood coagulation after cardiac arrest is not balanced adequately by activation of endogenous fibrinolysis," Circulation, vol. 92, no. 9, pp. 2572-2578, 1995.

[62] M. Fischer and K. A. Hossmann, "No-reflow after cardiac arrest," Intensive Care Medicine, vol. 21, no. 2, pp. 132-141, 1995.

[63] F. Spohr and B. W. Bottiger, "Safety of thrombolysis during cardiopulmonary resuscitation," Drug Safety, vol. 26, no. 6, pp. 367-379, 2003.

[64] P. A. Tiffany, M. Schultz, and H. Stueven, "Bolus thrombolytic infusions during CPR for patients with refractory arrest rhythms: outcome of a case series," Annals of Emergency Medicine, vol. 31, no. 1, pp. 124-126, 1998.

[65] B. W. Bottiger, C. Bode, S. Kern et al., "Efficacy and safety of thrombolytic therapy after initially unsuccessful cardiopulmonary resuscitation: a prospective clinical trial," The Lancet, vol. 357, no. 9268, pp. 1583-1585, 2001.

[66] I. Kurkciyan, G. Meron, F. Sterz et al., "Pulmonary embolism as cause of cardiac arrest: presentation and outcome," Archives of Internal Medicine, vol. 160, no. 10, pp. 1529-1535, 2000.

[67] K. Janata, M. Holzer, I. Kurkciyan et al., "Major bleeding complications in cardiopulmonary resuscitation: the place of thrombolytic therapy in cardiac arrest due to massive pulmonary embolism," Resuscitation, vol. 57, no. 1, pp. 49-55, 2003.

[68] W. Lederer, C. Lichtenberger, C. Pechlaner, G. Kroesen, and M. Baubin, "Recombinant tissue plasminogen activator during cardiopulmonary resuscitation in 108 patients with out-ofhospital cardiac arrest," Resuscitation, vol. 50, no. 1, pp. 71-76, 2001.

[69] W. P. Bozeman, D. M. Kleiner, and K. L. Ferguson, "Empiric tenecteplase is associated with increased return of spontaneous circulation and short term survival in cardiac arrest patients unresponsive to standard interventions," Resuscitation, vol. 69, no. 3, pp. 399-406, 2006.

[70] K. H. Stadlbauer, A. C. Krismer, H. R. Arntz et al., "Effects of thrombolysis during out-of-hospital cardiopulmonary resuscitation," American Journal of Cardiology, vol. 97, no. 3, pp. 305-308, 2006.

[71] D. M. Fatovich, G. J. Dobb, and R. A. Clugston, "A pilot randomised trial of thrombolysis in cardiac arrest (The TICA trial)," Resuscitation, vol. 61, no. 3, pp. 309-313, 2004.

[72] J. Gramann, P. Lange Braun, T. Bodemann et al., "Use of thrombolytics during resuscitation as a therapy of last resort in cardiac arrest," Intensiv-und Notfallbehandlung, vol. 16, pp. 134-137, 1991 (German).

[73] R. B. Abu-Laban, J. M. Christenson, G. D. Innes et al., "Tissue plasminogen activator in cardiac arrest with pulseless electrical activity," The New England Journal of Medicine, vol. 346, no. 20, pp. 1522-1528, 2002.

[74] B. W. Bottiger, H. R. Arntz, D. A. Chamberlain et al., "Thrombolysis during resuscitation for out-of-hospital cardiac arrest," The New England Journal of Medicine, vol. 359, no. 25, pp. 2651-2662, 2008.

[75] B. W. Bottiger and E. Martin, "Thrombolytic therapy during cardiopulmonary resuscitation and the role of coagulation activation after cardiac arrest," Current Opinion in Critical Care, vol. 7, no. 3, pp. 176-183, 2001.

[76] C. Adrie, M. Adib-Conquy, I. Laurent et al., "Successful cardiopulmonary resuscitation after cardiac arrest as a "sepsislike" syndrome," Circulation, vol. 106, no. 5, pp. 562-568, 2002. 
[77] G. Hekimian, T. Baugnon, M. Thuong et al., "Cortisol levels and adrenal reserve after successful cardiac arrest resuscitation," Shock, vol. 22, no. 2, pp. 116-119, 2004.

[78] V. A. Negovsky, "The second step in resuscitation: the treatment of the "postresuscitation disease"," Resuscitation, vol. 1, no. 1, pp. 1-7, 1972.

[79] D. Annane, E. Bellissant, V. Sebille et al., "Impaired pressor sensitivity to noradrenaline in septic shock patients with and without impaired adrenal function reserve," British Journal of Clinical Pharmacology, vol. 46, pp. 589-597, 1998.

[80] H. Prigent, V. Maxime, and D. Annane, "Clinical review: corticotherapy in sepsis," Critical Care, vol. 8, no. 2, pp. 122-129, 2004.

[81] P. E. Marik and G. P. Zaloga, "Adrenal insufficiency in the critically III: a new look at an old problem," Chest, vol. 122, no. 5, pp. 1784-1796, 2002.

[82] T. Ito, D. Saitoh, A. Takasu, T. Kiyozumi, T. Sakamoto, and Y. Okada, "Serum cortisol as a predictive marker of the out-come in patients resuscitated after cardiopulmonary arrest," Resuscitation, vol. 62, no. 1, pp. 55-60, 2004.

[83] C. H. Schultz, E. P. Rivers, C. S. Feldkamp et al., "A characterization of hypothalamic-pituitary-adrenal axis function during and after human cardiac arrest," Critical Care Medicine, vol. 21, no. 9, pp. 1339-1347, 1993.

[84] K. H. Lindner, H. U. Strohmenger, H. Ensinger, W. D. Hetzel, F. W. Ahnefeld, and M. Georgieff, "Stress hormone response during and after cardiopulmonary resuscitation," Anesthesiology, vol. 77, no. 4, pp. 662-668, 1992.

[85] M. Tsai, C. Huang, W. Chang et al., "The effect of hydrocortisone on the outcome of out-of-hospital cardiac arrest patients: a pilot study," American Journal of Emergency Medicine, vol. 25, no. 3, pp. 318-325, 2007. 


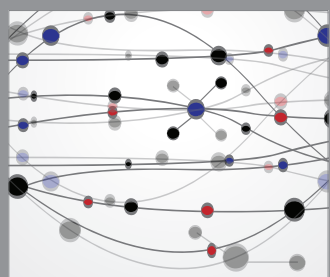

The Scientific World Journal
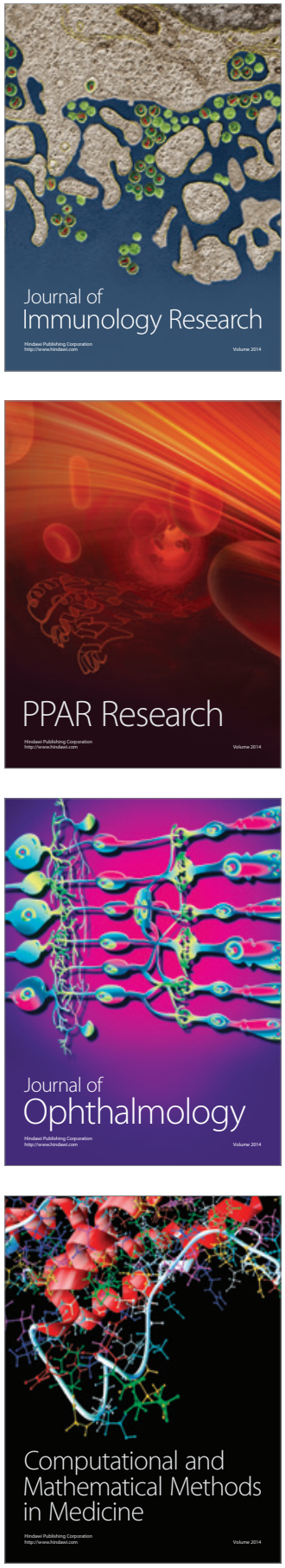

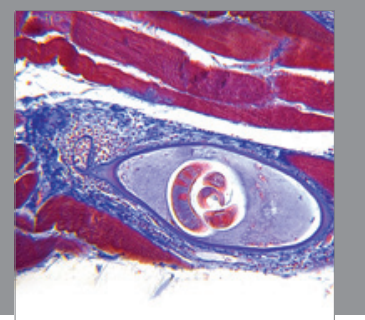

Gastroenterology

Research and Practice
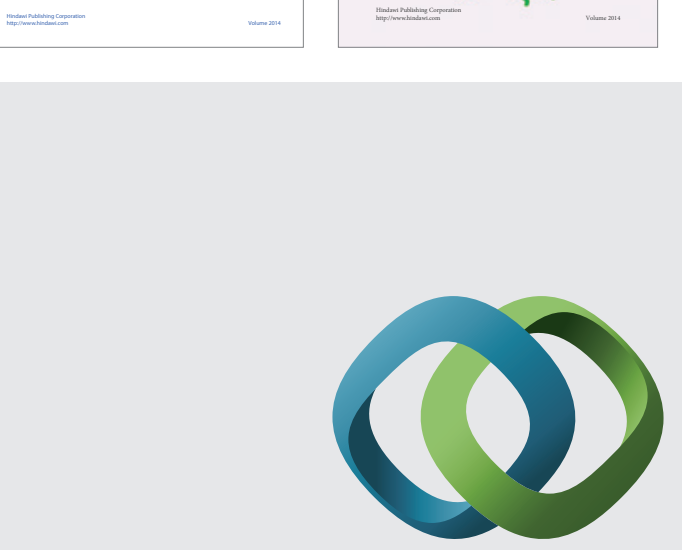

\section{Hindawi}

Submit your manuscripts at

http://www.hindawi.com
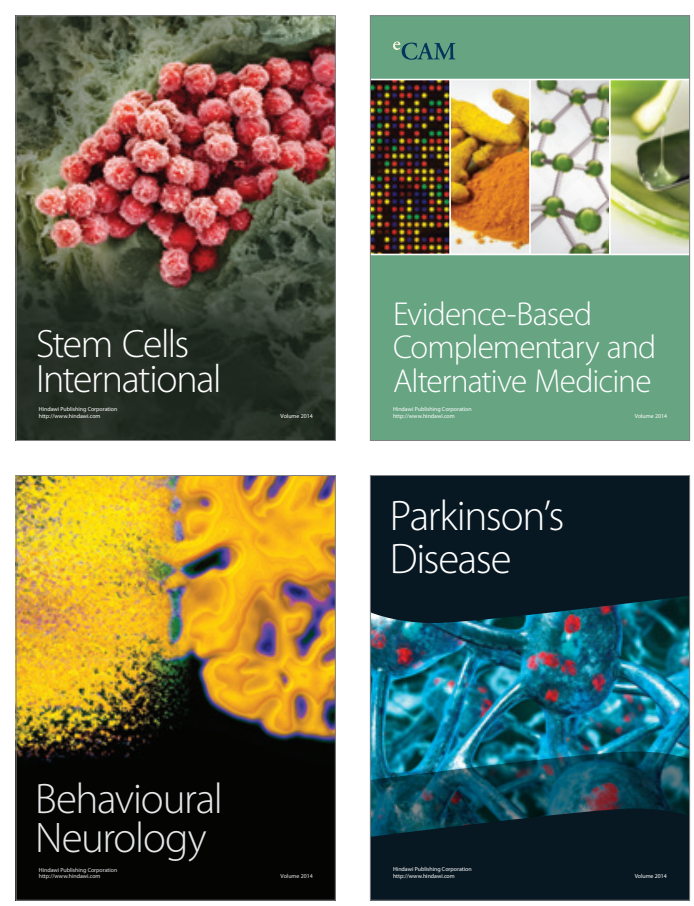

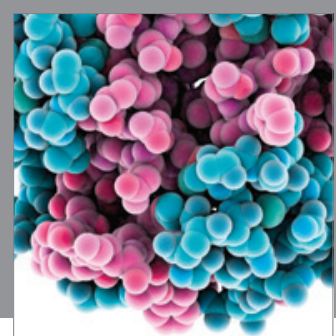

Journal of
Diabetes Research

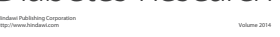

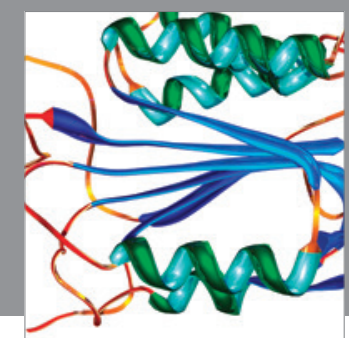

Disease Markers
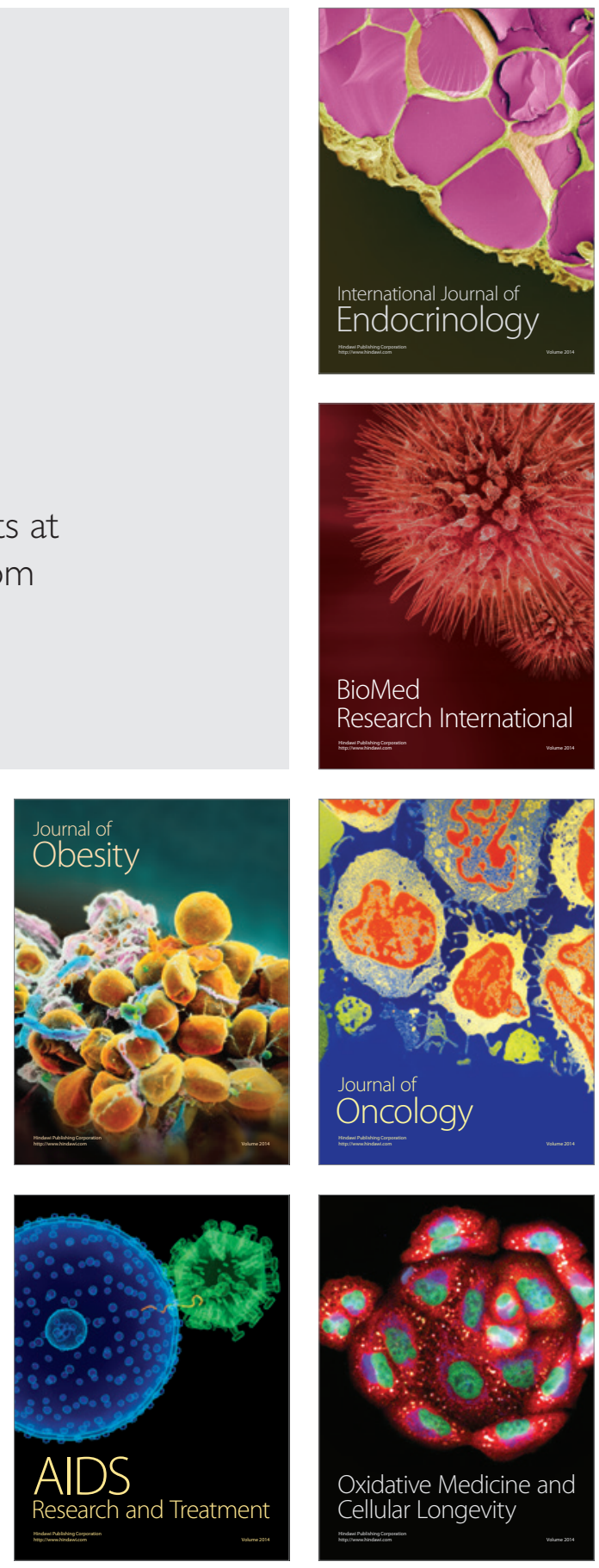